\title{
TERRITORIALIDAD COOPERATIVA Y CAMPESINA DEL MOVIMIENTO DE LOS TRABAJADORES RURALES SIN TIERRA (MST) DE BRASIL ${ }^{1}$
}

\author{
Samuel Ortiz Pérez $z^{2}$ \\ Dpto. Geografía Humana. Universidad de Alicante \\ samuel.ortiz@ua.es
}

\section{RESUMEN}

En enero de 1984, en el municipio de Cascavel, situado en la región más occidental del estado de Paraná (Brasil), se constituyó formalmente el Movimiento de los trabajadores rurales Sin Tierra, el MST. Su relevancia geográfica, histórica, económica y social queda cristalizada en su dinámica de territorialización, protagonizada por los asentamientos de reforma agraria, las cooperativas de producción y comercialización, y sus agroindustrias, así como por sus escuelas de formación. Se potencia con todo ello la defensa y viabilidad de una agricultura familiar y campesina, asentada en la práctica de la agroecología. El devenir de este proceso conforma un fenómeno geográfico de extraordinaria magnitud hacia la producción de una alternativa social y espacial.

Palabras clave: Cooperativas, agroecología, campesinado, alternativa espacial.

\section{ABSTRACT}

\section{Cooperative and peasant territoriality Brazilian Landless Rural Workers Movement (MST).}

In January 1984, in the town of Cascavel, located in the westernmost region of the state of Paraná (Brazil), the Brazilian Landless Rural Workers Movement (MST) was formally established. Its geographical, historical, economic and social relevance is crystallized in its territorial dynamics, led by the agrarian reform settlements, cooperative production and marketing, and agribusiness, as well as its training schools. It is enhanced by all the defense and viability of family and peasant agriculture, based on the practice of agro ecology. The evolution of this process forms a geographical phenomenon of extraordinary magnitude towards the production of a social and spatial alternative.

Keywords: Cooperatives, agroecology, peasantry, MST, alternative space.

\section{INTRODUCCIÓN}

El Movimiento de los trabajadores rurales Sin Tierra de Brasil (MST) se ha convertido en los últimos años en uno de los movimientos sociales más relevantes del país y, por tanto, del mundo (Harnecker, 2002). La lucha de los Sin Tierra constituye un movimiento popular de masas que recoge una serie de reivindicaciones históricas vinculadas, entre otras, a las demandas de una reforma agraria, la redistribución de tierras y la justicia social.

Los estudios y tesis doctorales sobre el MST, no sólo en Brasil, se han reproducido con mayor intensidad en la última década con motivo de su transcendencia social, política, económica, territorial e, incluso,

1 Este artículo ha sido realizado gracias a una Beca de Personal Investigador de carácter pre-doctoral concedida por la Consellería d’Educació de la Generalitat Valenciana, resolución del 27 de marzo de 2009 (BFPI2009/180).

2 Profesor del Departamento de Geografía Humana de la Universidad de Alicante. Miembro del Grupo de Investigación "Cooperativismo, Desarrollo Rural y Emprendimientos Solidarios en la Unión Europea y Latinoamérica" -COODRESUEL-: http://web.ua.es/es/ cooperativismo-ue-latinoamerical 
ideológica para la historia del campo brasileño (Carballo, 2011; Giribet Bernat, 2012). No obstante, y desde una perspectiva geográfica, la materialización de una organización cooperativa en el conjunto del proceso de producción supone el desarrollo de nuevas relaciones técnicas y sociales de trabajo. Así como diferentes formas de interacción con la naturaleza que derivan de la práctica real de un trabajo cooperativo tanto en la esfera de la producción como en la del consumo. A ello cabe añadir la defensa del MST $(2005,2008 a)$ por el desarrollo de una agricultura basada de manera exclusiva en las técnicas agroecológicas, el reconocimiento social de la identidad y forma de vida campesina, y la construcción de escuelas de formación técnica y política para su base social, acompañadas de un discurso transformador de la sociedad (Caldart, 2004).

Según datos publicados en el VI Congreso Nacional celebrado en Brasilia en febrero de 2014, el MST está presente en veinticuatro de los veintiséis estados del país y aglutina a unas 350.000 familias assentadas y 90.000 familias acampadas, que representan en torno a un millón de personas. Existen assentamentos o campamentos vinculados al Movimiento Sin Tierra localizados en 1.182 municipios de todo Brasil (21,23\%), y los assentamentos ocupan cerca de ocho millones de hectáreas. El MST cuenta aproximadamente con un centenar de cooperativas de producción activas en todo el país, de las cuales ocho son Cooperativas de Producción Agrícola consolidadas (sobre todo en la región sur), cinco cooperativas de crédito, a las que cabe sumar unas dos mil asociaciones de producción y servicios. Estas prácticas de trabajo cooperativo pueden darse tanto en la esfera de la producción, de la distribución o incluso en la organización propia de la vida social (finanzas, servicios, vivienda), cuyo énfasis sustenta el propio proyecto social y político de este movimiento socioterritorial (Fernandes, 2005) hacia una territorialización cooperativa y campesina del movimiento.

Por consiguiente, este estudio versa sobre las implicaciones socioterritoriales derivadas del estrecho vínculo hallado entre la organización social y política del MST y de su estructura económica-productiva, basada en la producción y comercialización de productos de reforma agraria a través de la creación de un sistema de cooperativas populares y de su distribución en Brigadas, las cuales han sido especialmente fomentadas por los Sin Tierra en el estado de Paraná, y la región sur de Brasil.

La realización de este trabajo se sustenta en la aplicación de encuestas y entrevistas a todas las cooperativas del MST del estado de Paraná existentes hasta el primer trimestre del año 2013 (muestra de un 100\%), con el objetivo de establecer un diagnóstico acerca de la base socioeconómica y territorial de sus cooperativas, así como de sus productos y canales de comercialización. En total, del conjunto de las cooperativas investigadas, 15 están consideradas Cooperativas de Producción y Prestación de Servicios (CPPS) de carácter regional; dos como Cooperativas de Producción y Servicios (CPS) de actuación básicamente local; una Cooperativa de Producción Agropecuaria (CPA); una Cooperativa de Crédito y, por último, una Cooperativa Central (CCA) que integraría al conjunto de cooperativas del estado Paraná, a modo de cooperativa de segundo grado. A esta evaluación final se adjunta la Asociación de Producción y Comercialización Irmã Dorothy (APROCID), localizada en el assentamento "Guanabara" en el municipio de Imbaú, motivado por su extraordinaria distribución del trabajo colectivo y ligada a la cooperativa de producción de semillas criollas COOPERTERRA (municipio de Ortigueira), que ostenta la particularidad de estar gestionada por un colectivo de mujeres campesinas. Además, el análisis ha sido completado con la participación en encuentros, congresos y reuniones sectoriales del MST, con la consecuente obtención de entrevistas con aquellas personas coordinadoras del Sector de Producción y Comercialización; con responsables de la Confederación de Cooperativas de Reforma Agraria de Brasil (CONCRAB), y también con el Grupo de Estudios Agrarios del MST (GEA)3.

Finalmente, las visitas a siete centros educativos y escuelas de formación propias del MST, distribuidas por todo el estado de Paraná, han facilitado de forma considerable el acceso a una amplia diversidad bibliográfica temática y a otra documentación de carácter interno, elaborada por el propio por el MST, así como por la CONCRAB. De igual manera, cabe destacar la oportunidad de participar en distintos cursos y debates (particularmente sobre agroecología, cooperativismo, y economía solidaria), así como la convivencia y el acompañamiento del Movimiento Sin Tierra, su mística y sus espacios de trabajo y de intercambio educativo y cultural, lo cual ha enriquecido el conocimiento acerca del movimiento y sus improntas socioterritoriales a través de sus cooperativas y sus escuelas.

3 Las personas entrevistadas fueron: Miranda, BA, Paulinha, Neuri Rossetto, Elmar, Pedro Christoffoli y Salette. 


\section{EL "SISTEMA COOPERATIVISTA DOS ASSENTADOS" DEL MST}

El Sistema Cooperativista dos Assentados (SCA) define un proyecto de organización cooperativa que garantice la viabilidad de la agricultura familiar campesina en los assentamentos de reforma agraria del MST, convirtiéndose así en un sector estratégico para la transformación de la vida en el campo y de la sociedad en su conjunto. Una transformación fundamentada en la generalización del trabajo cooperativo desde diferentes niveles y perspectivas.

Fue en el V Encuentro Nacional del MST, celebrado en el año 1989, cuando se determina uno de los lemas más representativos del movimiento: "Ocupar, Resistir y Producir", y a partir de esta proclama se abrió el camino para la creación del llamado Sistema Cooperativista dos Assentados (SCA). Esta propuesta de organización y estructura cooperativa ostenta, en términos generales, un ideario organizativo hacia la consolidación de un proceso de cooperación agrícola para las familias asentadas en la lucha por la reforma agraria en el país, donde se recogen diversas prácticas de distintas iniciativas de cooperación agraria provenientes de la experiencia ocurrida tanto en el movimiento campesino brasileño como en otras de carácter internacional.

La práctica cooperativista del MST integra diferentes niveles de cooperación. Por tanto, en los campamentos y assentamentos del MST existe una gran variedad de formas de cooperación que pasamos a identificar, y que engloba desde aquellas más simples hasta otras más complejas en cuanto a su nivel de intensidad, estructura y dimensión. Entre las formas simples de cooperación constan las prácticas de ayuda mutua y solidaridad en el trabajo (por ejemplo, 'o mutirão' y 'o puxirão'), que consiste en llevar a cabo intercambios de servicios o tareas puntuales ejercidas de forma colectiva como plantar, rozar, recoger la cosecha, sacar la leche de las vacas; para el mantenimiento y limpieza de las infraestructuras, la organización y preparación de comidas colectivas, entre otras prácticas comúnmente presentes en la vida cotidiana campesina. De esta manera, las familias de diferentes lotes de tierra se unen en Núcleos de Producción (NP), establecidos según criterios de proximidad o bien por participar de una misma línea de producción, con la finalidad de colaborar en el proceso de trabajo ${ }^{4}$.

Las diversas prácticas de cooperación y ayuda mutua se organizan también mediante la creación de grupos colectivos o semicolectivos, de carácter informal, con la finalidad de cooperar en tareas de producción, comercialización y otros objetivos comunes. Se trata, pues, de iniciativas impulsadas por familias asentadas en las que cada una de ellas aporta una parte de su tierra (o toda su parte) para trabajarla colectivamente, y con ello producir y vender la cosecha de forma conjunta. Entre otras formas simples de cooperación quedarían incluidas las asociaciones en los campamentos y assentamentos, que se encargan de ejecutar varias tareas de forma asociativa: la adquisición conjunta de animales (vacas lecheras, ganado, cerdos, o gallinas camperas para la producción de carne y huevos, estanques para peces...); la compra de insumos agrícolas y maquinaria (tractores, segadora, sistemas de irrigación...), y de medios de transporte (camioneta o combi, un autobús, una furgoneta, un coche o motocicleta...); la construcción de nuevos espacios productivos (un almacén, una casa de harina, una agroindustria, una tienda...), la optimización de una asistencia técnica, la búsqueda de nuevos mercados, entre otras.

Sin embargo, las asociaciones también cumplen otro papel complementario en los assentamentos de reforma agraria. Es decir, funcionan como instrumento jurídico de representación de las familias con el objetivo principal de facilitar las tramitaciones burocráticas de aquellos servicios públicos y recursos gubernamentales destinados a la mejora de las condiciones de vida del conjunto de familias asentadas. A través de estas asociaciones se canaliza la presentación de proyectos públicos (educación, vivienda, energía, sanidad, transporte, medio ambiente) y las solicitudes de proyectos económicos destinados a la organización de la producción y comercialización de sus productos. En gran medida, éstas quedan supeditadas a la estructura socio-política principal de assentamentos y campamentos, la "Coordinación del Assentamento" (o bien del campamento), instancia donde están representadas las familias, organizadas en Núcleos de Base, y los sectores de actividad. Aquí es donde, en definitiva, se toman las principales decisiones geoestratégicas que afectan al conjunto del territorio adquirido 5 .

4 Los Núcleos de Producción (organización económico-productiva) pueden coincidir con los Núcleos de Base (organización política), aunque no siempre.

5 Las asociaciones complementan la estructura política del assentamento y campamento como mecanismo legal y con personalidad jurídica registrada. 
Entre las formas complejas de cooperación estarían los diferentes tipos de cooperativas creadas por el Movimiento Sin Tierra. En primer lugar se hallan las Cooperativas de Prestación de Servicios (CPS). Son aquellas formadas por personas de un mismo assentamento, encargadas de la asistencia técnica y la dotación de insumos a las familias asentadas y, si fuera el caso, de la distribución de los productos generados por los propios Núcleos de Producción, por los grupos colectivos y semicolectivos, o bien por las asociaciones. Pero cuando estas cooperativas se estructuran para alcanzar un mayor número de familias, aglutinando a dos o más assentamentos y campamentos, normalmente localizados en varios municipios cercanos de una misma región, se constituyen las Cooperativas de Prestación de Servicios Regionales (CPSR). Éstas actúan a una escala mayor, lo que permite implantar procesos de transformación y manipulación de productos primarios (beneficiamento) mediante la instalación de una o varias agroindustrias, con la obtención de un mayor valor añadido en los productos finales elaborados para su comercialización.

Por contra, esta modalidad de cooperativas regionales, más propia de la primera etapa cooperativista del MST, que alberga hasta finales de los años noventa, ha sido progresivamente sustituida por la constitución de Cooperativas de Producción y Prestación de Servicios (CPPS), ligadas a la nueva propuesta de organización política y territorial del MST implantada a lo largo de la primera década del presente siglo. Así, las CPPS se estructuran a una escala territorial supramunicipal, integrando a varios assentamentos y campamentos en una misma Brigada. En estas cooperativas distribuidas por Brigadas (organización social y política), se procede a una mejora sustancial en la planificación del proceso productivo, lo que permite la manipulación y transformación de sus productos a través de la instalación de pequeñas agroindustrias, una mayor disponibilidad de asistencia técnica y el acceso a insumos y materiales de trabajo, así como la apertura de nuevos canales de comercialización para las diferentes líneas de producción, todo ello mediante una gestión cooperativa.

Es preciso señalar que la base social de estas cooperativas corresponde en su mayoría con las familias asentadas en lotes individuales junto con otras familias acampadas, todas ellas ligadas al movimiento Sin Tierra aunque no de forma exclusiva, las cuales aportan su producción a la misma. Este tipo de cooperativa adquiere y concentra la producción de toda su base social generando un nuevo producto transformado y se responsabiliza de su distribución y comercialización. Las CPPS se encargan a su vez del seguimiento de todo el proceso de trabajo en el conjunto de la cadena productiva. Las familias reciben un "retorno del excedente" (en forma de dinero) según su actividad cooperativizada, es decir, la actividad aportada a la cooperativa, que puede ser medida bien por las horas empleadas de trabajo en la cooperativa ("ayuda de coste"), o bien según la cantidad y calidad de la producción aportada (litros de leche, kilos de arroz, de frutas, hortalizas, miel, etcétera). El control del conjunto de la cadena productiva por parte de una CPPS garantiza, por un lado, una completa seguridad de venta de la producción de las familias assentadas y una cierta estabilidad económica, obteniendo generalmente un mejor precio por unidad de producción, y por otro lado, la posibilidad de generar una mayor diversidad de productos a través de sus agroindustrias, al mismo tiempo que la agregación de valor al producto final. A lo que cabe añadir la posterior distribución cooperativa y la transcendente difusión de los productos de la agricultura familiar (con la marca MST), que repercute directa o indirectamente en el aumento de la renta de las familias campesinas asentadas del movimiento y la mejora de la calidad de vida de los assentamentos de reforma agraria.

En otro nivel de complejidad de la organización del trabajo cooperativo se sitúan las Cooperativas de Producción Agropecuaria (CPA), considerada como una etapa superior de organización de la producción y de trabajo colectivo (Concrab, 1998; Harnecker, 2002). En este tipo de cooperativa la principal característica es que el trabajo y la propiedad de los medios de producción, especialmente la tierra, es totalmente colectiva. Se trata de una "propiedad social, donde los trabajadores son los dueños, y de una producción social, donde los trabajadores se reparten los excedentes de acuerdo a la aportación de trabajo de cada uno" (MST, 2008: 68). Asimismo, las CPA del Movimiento Sin Tierra representan un modelo de organización productiva de gran complejidad y un desafío de primer orden en la producción de una alternativa social y espacial. Ello supone la práctica de un trabajo cooperativo real y la participación de su base social en cada una de las fases de la cadena productiva, que incluye la esfera de la producción, industrialización, comercialización, administración y coordinación de la misma. Un propuesta que se extiende, además, en la implantación de un trabajo cooperativo y de relaciones de ayuda mutua en la gestión social y política del territorio.

6 Las "Brigadas del MST" se consolidan a principios del siglo XXI como una nueva forma de organización política, social y productiva para el movimiento, con una proyección territorial de extraordinaria relevancia en el estado de Paraná (ver apartado 3). 
Toda esta heterogeneidad de cooperativas se integra en una Cooperativa Central de Reforma Agraria (CCA), equivalente, grosso modo, a una cooperativa de segundo grado, es decir, una cooperativa de cooperativas. De manera que todas las cooperativas de un mismo estado se articulan mediante la CCA/estadual ${ }^{7}$ correspondiente. Éstas tienen la función de representar a todas las cooperativas del MST existentes en su territorio y son las responsables de llevar a cabo una función política y económica a favor de sus propias cooperativas. De igual modo, se hallan las cooperativas de crédito gestionadas por el MST, fundamentalmente presentes en la región sur del país, creadas con la finalidad geoestratégica de facilitar el acceso al crédito, las inversiones colectivas y los seguros para las familias campesinas asentadas en los assentamentos de reforma agraria, cuya función se sustenta en una serie de criterios éticos y de acción comprometida con el objetivo de rescatar el papel social del dinero.

La articulación territorial del sistema cooperativista del MST se completa con la creación en mayo de 1992 de la Confederación de las Cooperativas de Reforma Agraria de Brasil (CONCRAB), encargada de formar y fomentar la cooperación agrícola en todos los assentamentos y campamentos de los Sin Tierra, una instancia donde se coordinan las Cooperativas Centrales de Reforma Agraria de cada estado.

Figura 1. Territorialización del trabajo cooperativo en el MST.
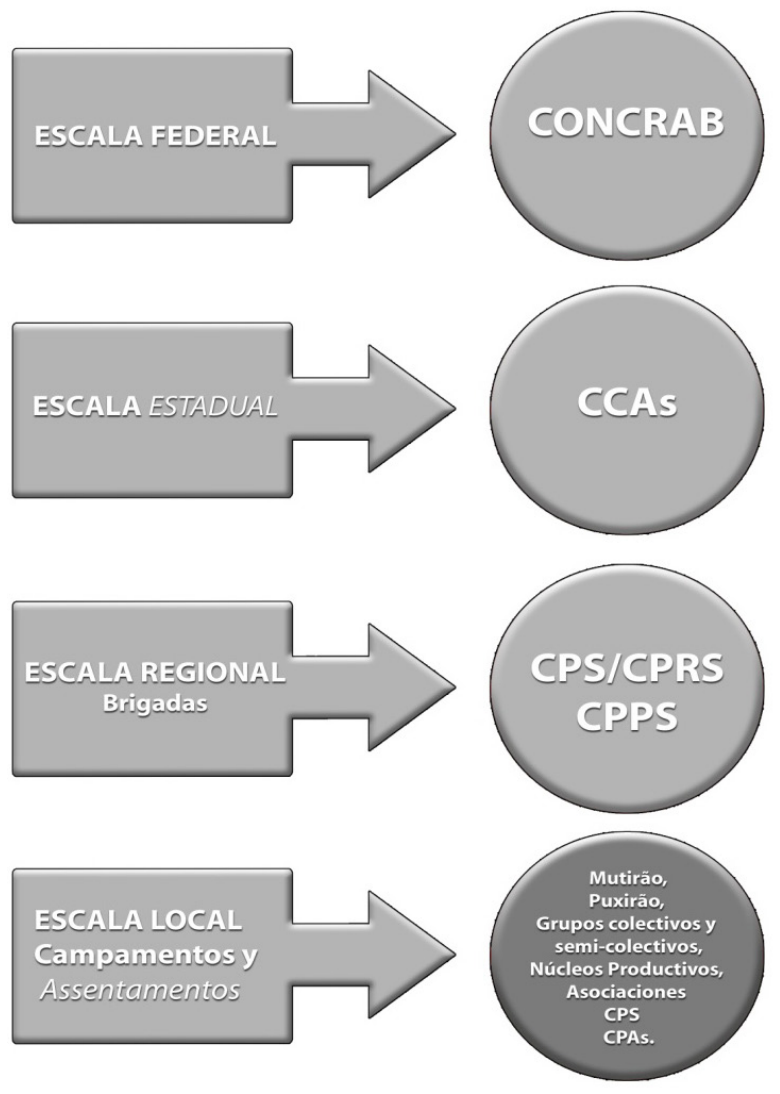

Elaboración propia

Entre los objetivos de la Concrab $^{8}$ se señala la superación de las contradicciones de las cooperativas, la consolidación y difusión de la cooperación agrícola, así como la mejora del trabajo cooperativo y co-

7 CCA/estadua l (en portugués) hace referencia a la Cooperativa Central de Reforma Agraria, en este caso de estudio, la del estado de Paraná.

8 En el año 2003, la CONCRAB promovió un Programa de Acompañamiento a las Empresas Sociales (PAES) del MST con el objetivo de implantar distintas formas de cooperación entre familias campesinas asentadas, con la finalidad de revalorizar el trabajo cooperativo en todas las áreas de reforma agraria. El informe definitivo fue publicado en mayo de 2008 en el cual se detallan las tareas realizadas por el equipo técnico, que incluían un diagnóstico de las empresas sociales (cooperativas y también de asociaciones), un estudio sobre el plan de actuación y planificación de aquellas tareas que se precisara, pasos para impulsar proyectos de agroindustria, de producción agropecuaria y de crédito, y, por último, la promoción de la empresa social y el fomento de nuevas empresas asociativas en assentamentos. 
lectivo en los assentamentos de reforma agraria (grupos de trabajo colectivo, asociaciones y cooperativas). Este proceso de territorialización cooperativa se fundamenta en: (1) la gestión de la comercialización de los productos de reforma agraria, para lo cual se propone la creación e instalación de agroindustrias y el control completo de la cadena productiva por parte de las propias cooperativas; (2) aumentar la productividad y la eficiencia técnica del trabajo cooperativo; (3) avanzar sobre las limitaciones tecnológicas, de modernización y capitalización de la producción; (4) la integración de la juventud en el trabajo de la agricultura familiar colectiva; (5) la consecución de una mejora sustancial de la renta familiar agregando valor a los productos de reforma agraria; (6) la rotación de los cargos de responsabilidad y de trabajo orientados por la cooperativa, con la finalidad de integrar el trabajo y aprender las funciones de las diferentes áreas productivas de la misma e identificarse con el trabajo colectivo realizado, y (7) facilitar el acceso de una financiación económica para los emprendimientos de reforma agraria con la obtención de créditos que permitan una viabilidad suficiente de los proyectos cooperativos.

En última instancia cabe hacer mención a la organización territorial de los assentamentos de reforma agraria que influye considerablemente en la estructura socioterritorial de las cooperativas del MST. Existen, de manera general, tres tipos de assentamentos que presentan diferentes formas de territorialización y de organización de las familias asentadas. El más común entre ellos es el tipo de assentamento distribuido en parcelas familiares individuales ${ }^{9}$, divididas en explotaciones familiares en usufructo, donde la producción y comercialización queda bajo la responsabilidad de cada una de las familias particulares asentadas, que pueden estar o no integradas en un grupo de trabajo colectivo o semicolectivo, una asociación o cooperativa.

Otro tipo de distribución es aquel de carácter colectivo, donde la distribución de la tierra y de los medios de producción es completamente colectiva, y cuya estructura productiva y comercial se coordina y planifica a través de una cooperativa, preferentemente una Cooperativa de Producción Agropecuaria (CPA). Es el caso de la Cooperativa de Producción Agropecuaria Victoria Ltda. (COPAVI), en el Estado de Paraná, o bien de la Cooperunião, en el assentamento "Tracutinga", en el Estado de Santa Catarina. Pero, no siempre todas las CPA presentan una organización totalmente colectiva en el assentamento, ni integran a todas las familias del mismo ${ }^{10}$.

Entre ambos modelos existen, no obstante, diversas formas híbridas de organización social y territorial de los assentamentos, considerados mixtos. En ellos se combinan tareas individuales con otras colectivas de producción y comercialización (grupos, núcleos, asociaciones). Por ejemplo, las cooperativas regionales (CPPS) integran tanto a personas que desarrollan una actividad individual o familiar como a otras organizadas en grupos informales de trabajo cooperativo. Por tanto, en la gran mayoría de assentamentos y campamentos coexiste una rica variedad de combinaciones de trabajo individual y colectivo, siendo esta última, la consolidación y generalización de prácticas de trabajo cooperativo y estructuras cooperativas, una prioridad para el movimiento en todas sus manifestaciones cotidianas. En este sentido se puede apreciar que, aunque no en todos los estados donde está organizado el MST existen cooperativas en su forma más compleja (tipo CPA o CPPS), prácticamente en todos los assentamentos y campamentos del MST existen numerosas formas de cooperación y prácticas de ayuda mutua.

\section{UNA TERRITORIALIZACIÓN COOPERATIVA EN EL ESTADO DE PARANÁ}

A partir de los criterios establecidos para la configuración del Sistema Cooperativista dos Assentados y la constitución de la CONCRAB como estructuras de referencia en la articulación de la producción cooperativa para la base social del movimiento, se crearon y desarrollaron las primeras cooperativas del MST. El objetivo primordial era (y es), por tanto, demostrar la viabilidad socioeconómica de los assentamentos de reforma agraria y revelar que mediante el trabajo cooperativo es posible mejorar de forma sustancial las condiciones de vida de las familias campesinas asentadas, superando así la producción básica de subsistencia. La concepción de la cooperación para el MST destaca por la búsqueda de un desarrollo económico y social, de valores humanistas y socialistas, una cooperación que debe estar vinculada a un proyecto estra-

9 El MST denuncia que el INCRA, responsable de la planificación territorial de los assentamentos de reforma agraria (PA), siempre dispone una organización individual de los mismos, dificultando desde el principio una reestructuración del territorio en base a valores cooperativos. Incluso, en el 2013 se ha denunciado la intención por parte del Gobierno brasileño de "privatizar" los lotes de tierra distribuidos en la reforma agraria, dotándoles de un título de empoderamiento individual de la propiedad (Medida Provisional del Gobierno 636)

10 La prestigiosa Cooperativa de Produção Agropecuária dos Assentados de Tapes (COOPAT), en Rio Grande do Sul, no consiguió en sus inicios integrar a todas las familias del assentamento. Posteriormente y de forma progresiva más familias quisieron incorporarse al proyecto cooperativo 
tégico, que visualice un cambio de la sociedad, para lo cual se necesita organizar a los trabajadores, preparar y liberar cuadros, ser masiva; una cooperación de lucha y resistencia al capitalismo (Concrab, 1995: 9).

La perspectiva general de las primeras cooperativas fue de marcado carácter económico y enfocado fundamentalmente a la producción, en busca de "cooperativas ejemplares" que incrementasen esencialmente la actividad productiva. Al mismo tiempo, era preciso establecer nuevas relaciones sociales dentro y fuera del lote familiar, basadas en la práctica de un trabajo cooperativo en aras de un nuevo modelo de sociedad, englobadas en un proyecto político más ambicioso. Así, las CPA se presentaban como "espacios de resistencia y de lucha del movimiento frente a las ofensivas del gobierno" (MST, 2008: 85) y también frente a la expansión de una agricultura eminentemente capitalista, el denominado agronegocio ${ }^{11}$.

El MST impulsa, en consecuencia, la construcción de un cooperativismo alternativo al modo económico capitalista, demostrando a la sociedad que es posible organizar la economía sobre otras bases y valores (MST, 1998: 10). Así pues, con esta visión política (de clase) de la cooperación se crean durante los primeros años de la década de los noventa, unas cooperativas organizadas por lo general en un solo assentamento, es decir, a una escala pequeña y de ámbito local, es decir, se priorizaron las cooperativas tipo CPA como modelo de organización colectiva. Pocos años después se realiza una valoración general del proceso de cooperación agrícola instalado hasta el momento y se celebra un seminario temático sobre "Las perspectivas de la cooperación en el MST", en diciembre de 1994. Entre las conclusiones del mismo surgió la necesidad de constituir cooperativas de comercialización y de prestación de servicios que pudieran responsabilizarse de gestionar los excedentes de la producción a otra escala, y que se encargaran de realizar análisis económicos de mercado para valorar la potencialidad de una salida comercial de los productos de reforma agraria. De la misma forma se identifican otros problemas de la base social como la escasa capacidad de acceso al crédito por parte de las familias, la dificultad de abrir mercados, la necesidad de una mejor planificación de la producción, así como la obligación de avanzar en la organización del trabajo cooperativo, en la demanda de servicios, insumos y asistencia técnica para las familias.

Ante esta perspectiva se promueve la construcción de cooperativas con una nueva dimensión territorial. Una nueva estructura cooperativa representada por las CPRS y, posteriormente, las CPPS, que abarca una base social localizada en diversos assentamentos (aunque no necesariamente distintos municipios), al mismo tiempo que incluye a familias organizadas en núcleos, colectivos o asociaciones de campamentos del MST con disponibilidad de producir pero con una escasa capacidad técnica y financiera. Además, junto a ellos se añade la posibilidad de incluir en este proceso a otros pequeños productores locales no integrados precisamente en el MST ${ }^{12}$

En las postrimerías del siglo XX fueron determinantes algunas crisis relacionadas con cuestiones de financiación, endeudamiento, gestión, y dificultades serias de acceso al crédito que afectó a numerosas cooperativas del movimiento. Por una parte, el MST denuncia que durante esos años el Gobierno federal representado en la ocasión por Fernando Henrique Cardoso y los gobiernos estaduales conservadores diseñaron diferentes mecanismos burocráticos con el objetivo de imposibilitar el acceso al crédito y dificultar las oportunidades de inversión de las cooperativas del MST. Pero ello no fue óbice, por otra parte, para que en el seno del MST se iniciara un proceso de valoración crítica sobre su propio sistema de cooperación agrícola y de organización cooperativa empleado hasta el momento. Se celebraron, en consecuencia, diferentes encuentros entre 2001 y 2003 para analizar esta cuestión y debatir nuevas acciones estratégicas en relación a la articulación y participación de la base social del movimiento, planteando de esta forma pautas hacia la configuración de una nueva organicidade del $\mathrm{MST}^{13}$.

11 Se utiliza el término "Agribusiness" (traducido al español como "Agronegocio") para designar la implantación de un modelo neoliberal de producción agraria basado en la monocultura, para el abastecimiento de una agroindustria altamente capitalizada y de una potente capacidad tecnológica, cuyos productos van destinados, generalmente, a la exportación y comercialización con países terceros. El agronegocio hace referencia a la expansión y consolidación de una agricultura capitalista y capitalizada, contextualizada en la dinámica del capital que requiere de nuevos territorios de acumulación.

12 Existe una colaboración estrecha del MST con otras organizaciones campesinas que apoyan un mismo proyecto de reforma agraria como, por ejemplo, el Movimiento de los Pequeños Agricultores (MPA).

13 A partir del 2002 se desarrolla un "Mutirão, Vamos Ouvir a Nossa Base", que implica a todas las familias de los assentamentos en los debates internos y sitúa a su base social en el centro de las acciones y de los sectores estratégicos con vistas al diseño de una nueva estructura del movimiento (MST, 2008). Se desarrollan nuevas metodologías de trabajo aplicadas a su carácter de movimiento de masas con el fin de potenciar la participación de las familias en el devenir del MST. Entre los objetivos de este proceso se propone la ampliación de los espacios de participación de la base social; el aumento del nivel de conciencia política y cultural de las familias; la formación de militantes y cuadros para el movimiento; el establecimiento de un mayor control político del espacio geográfico; el refuerzo de las estructuras de coordinación interna, y el fortalecimiento de la planificación y organización de acciones para avanzar en la reforma agraria. 
En esta restructuración social y política del MST surgen las Brigadas como proyecto de organización socioterritorial, acompañado de una redefinición de todos los sectores del movimiento, que en el caso de la producción pasa a denominarse Sector de Producción, Cooperación y Medio Ambiente (SPCMA). Entre las metas de trabajo planteadas conjuntamente con la Dirección Nacional del MST se indica la importancia de incidir en la formación político-ideológica y técnica de su base social, en la constitución de nuevas experiencias de organización de los assentamentos en núcleos de base, en incentivar la cooperación en la fase de producción y en motivar cambios en la matriz técnica y tecnológica de la producción, sobre todo en la producción dedicada a las semillas criollas, con el objetivo de consolidar los valores y acciones vinculadas a la Agroecología.

No obstante, las Brigadas surgen a inicios de siglo XXI tras un proceso colectivo de debate dentro del movimiento; y por consiguiente, representan un "eje central (geoestratégico) de la nueva organicidade del MST en la lucha por la reforma agraria y la transformación de la sociedad" (MST, 2005: 110).

Figura 2. Territorialidad de las brigadas del MST. Estado de Paraná, 2013.

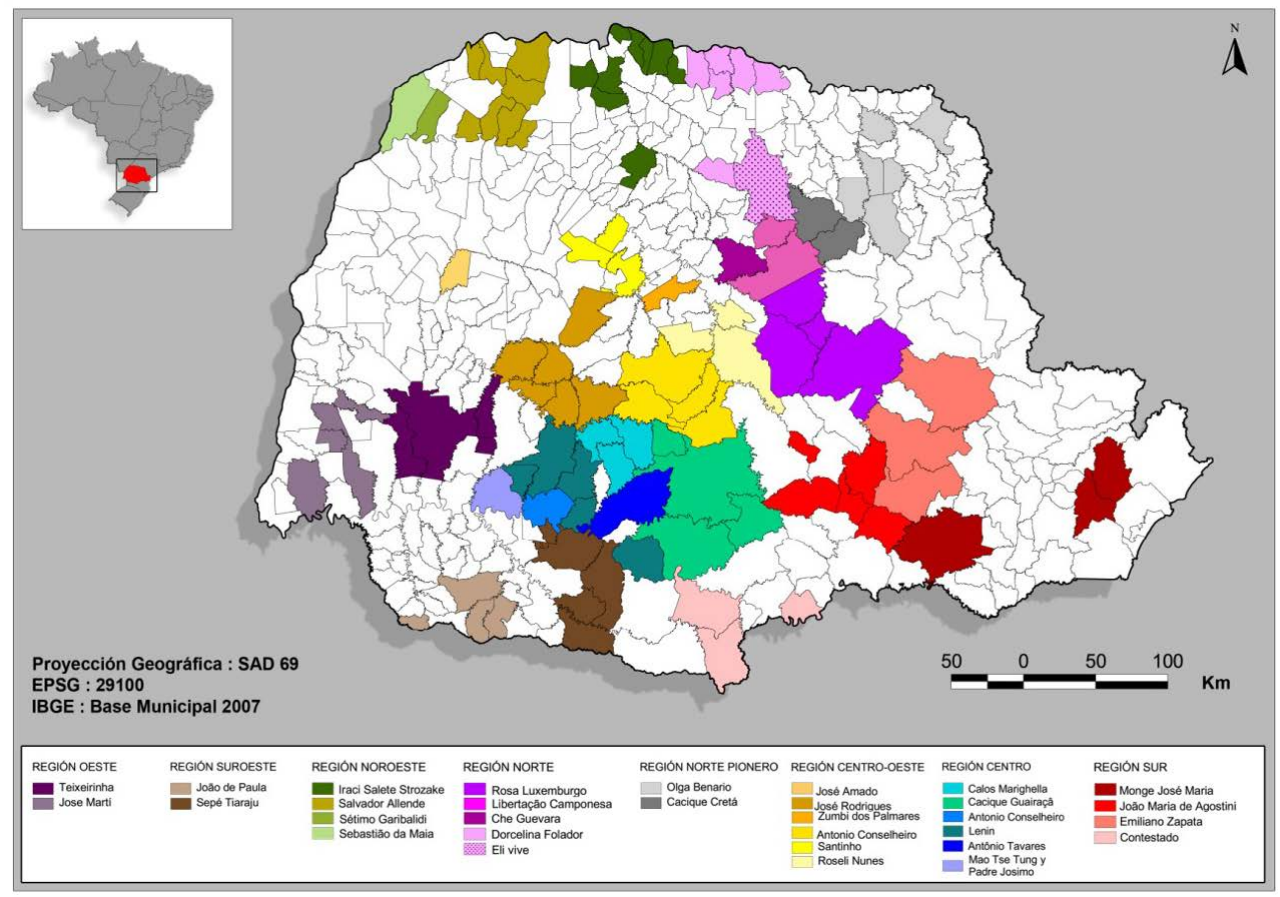

Elaboración propia

Nota: Actualización y ampliación de la versión MST-Paraná (Indianara C. Pires, 2010)

Bajo la responsabilidad del Sector de Producción del MST (SPCMA), las cooperativas se van articulando paulatinamente en relación a la territorialización de las Brigadas, con especial relevancia en el estado de Paraná. Cada brigada está compuesta por unas 500 familias procedentes de assentamentos y también de campamentos, estructuradas en 10 núcleos de 50 familias cada uno. La "Coordinación de la Brigada" tiene autonomía propia y está integrada por una persona de la dirección estadual, una persona coordinadora de cada núcleo de 50 familias, más aquellas representantes de los diferentes sectores creados en la brigada (formación, sanidad y género, frente de masas, producción, finanzas, comunicación, cultura, otros ${ }^{14}$. Y, a su vez, pueden formar parte de la misma dos personas (un hombre y una mujer) por cada núcleo de 10 familias, que pueden coincidir o no con otras responsabilidades. En resumen, se podría hablar de una Coordinación compuesta por un dirigente estadual, las representantes de cada sector y las personas coordinadoras de los núcleos de familias, que suman aproximadamente entre unas 175 y 200 personas en la coordinación de la brigada. Desde este nuevo marco organizativo se impulsan nuevas cooperativas y se rescatan algunas cooperativas inactivas. Esta regeneración supuso, sin embargo, la 
desestructuración de otras cooperativas antiguas que no pudieron hacer frente a las problemáticas tanto externas como internas en la organización del trabajo y de su viabilidad económica como, por ejemplo, la cooperativa COAGRI en la región centro del estado de Paraná.

Los documentos publicados por el MST desde sus inicios hasta el reciente VI Congreso del MST constatan que la cooperación no es simplemente concebida como un medio de trabajo, un modelo de desarrollo o un mero factor de mejora de las condiciones de vida de familias campesinas. La cooperación (no solo en el trabajo sino como práctica desarrollada en la vida social) es entendida como una dinámica territorial estratégica de transformación socioespacial, que precisa ser teorizada y materializada en sus diferentes variantes y en todos los ámbitos. De manera que las cooperativas del MST se encuentran inmersas en un programa político mayor y más ambicioso vinculado a los valores de una soberanía alimentaria ${ }^{15}$ y a la transformación de las relaciones de poder espacial, en un proyecto político integrado por valores humanistas y socialistas (ideológicos) y de conciencia de clase (formación y educación política y técnica).

He ahí donde radica la importancia dialéctica de la intercooperación entre cooperativas y del trabajo cooperativo en la producción de un nuevo espacio geográfico, no exenta por supuesto de contradicciones, ni aislada de la imposición espacial del capital.

\subsection{Distribución socioterritorial de las cooperativas paranaenses del MST}

El Estado de Paraná es uno de los estados brasileños con mayor tradición histórica del movimiento cooperativista y asociativo agrario del país. Es uno de los tres estados que componen la región sur de la República Federal de Brasil con un área de 199.307, 922 km² (algo menos de la mitad de la extensión que ocupa España), y donde habitan unas 10,9 millones de personas, según el Instituto Brasileño de Geografía y Estadística (IBGE, 2013). El movimiento cooperativo queda representado a nivel federal por la Organización de Cooperativas Brasileñas (OCB), que en Paraná suma un total de 240 cooperativas en el Sistema Ocepar ${ }^{16}$, de las cuales 81 son cooperativas agropecuarias, representando alrededor de un $55 \%$ de la economía agrícola del estado. Sin embargo, las cooperativas del MST no forman parte de este Sistema Ocepar, fundamentalmente por motivos ideológicos y políticos, por lo que sus cooperativas se encuentran federadas en la Confederación de Cooperativas de Reforma Agraria de Brasil (CONCRAB), y sobre las cuales la OCB no presenta datos estadísticos.

A finales del año 2013, el MST cuenta con unos 322 assentamentos y una decena de campamentos localizados en 126 municipios del estado de Paraná (de un total de 399 en el estado), integrados por unas 28.000 familias, y que conjuntamente albergan unas 420.000 hectáreas de tierra destinadas a la construcción de viviendas rurales, la planificación de una actividad campesina y familiar, y la instalación de pequeñas agroindustrias y escuelas. Asimismo destaca la existencia de más de ciento diez asociaciones de producción y unas veinte cooperativas de producción y comercialización distribuidas por todo el estado. De este modo, las cooperativas en el estado de Paraná se distribuyen territorialmente de acuerdo a la estructura social y política de las Brigadas del MST.

Las cooperativas se clasifican según la línea de producción principal y secundaria, estableciendo así una intercooperación sectorial entre cooperativas en el conjunto del movimiento bajo la coordinación general de la propia Cooperativa Central de Paraná (CCA), cuya sede central se localiza en la capital del estado, Curitiba. La base social de cada cooperativa se encuentra distribuida en diferentes assentamentos, y solo en algunas ocasiones en un único assentamento como en el caso de las CPS y más particularmente en las CPA. Las familias asentadas, organizadas en Núcleos de Producción, son las principales fuentes de trabajo, encargadas sobre todo de la fase de producción en su lote familiar, generalmente individualizado, aunque existen, como ya hemos señalado, distintas formas simples de cooperación en el proceso de trabajo (grupos colectivos y semicolectivos). Por otro lado, también existen personas asalariadas que están más

15 La producción espacial de una soberanía alimentaria se encuentra estrechamente relacionada con la producción de un nuevo espacio geográfico impulsado, fundamentalmente, desde los movimientos sociales del campo, cuya esencia radica en reproducir dinámicas socioterritoriales con base en una práctica no-capitalista. Esta práctica consistiría en que pueblos y comunidades, naciones y/o estados, adquieran el derecho básico a la alimentación mediante la autonomía territorial y el empoderamiento de sus decisiones políticas y de su organización económica, principalmente en todo aquello que se refiere a la esfera de la producción y el consumo de alimentos (HoltGimenez et al, 2008).

16 La Ocepar es la sección estadual de la Organizacion de Cooperativas Brasileñas (OCB) en el estado de Paraná, dedicada a la representación y el fomento del cooperativismo, así como a la capacitación y desarrollo de las cooperativas paranaenses. 
presentes en las fases de industrialización, asistencia técnica y distribución. Su remuneración como socias de la cooperativa se establece mediante una "ayuda de coste" como incentivo económico por el desempeño de tareas específicas que contribuyen al ejercicio del trabajo cooperativo. Se trata de personas con una especialización técnica y de una indispensable responsabilidad en el proceso productivo, apoyadas en ocasiones por el beneficio de políticas, recursos y organismos públicos (por ejemplo, ATER: Servicios de asistencia técnica y extensión rural).

Tabla 1. Estructura territorial de las cooperativas del MST por tipo, brigada y municipio. Estado de Paraná (Brasil), 2013.

\begin{tabular}{|c|c|c|c|}
\hline COOPERATIVA & Tipo & BRIGADA(s) & $\begin{array}{l}\text { Sede Central } \\
\text { Municipio }\end{array}$ \\
\hline CORAU & CPPS & Iraci Salete Stronzake & Paranacity \\
\hline COANA & CPPS & $\begin{array}{l}\text { Sebastião da Maia } \\
\text { Sétimo Garibaldi } \\
\text { Salvador Allende }\end{array}$ & Querência do Norte \\
\hline COANOP & CPPS & $\begin{array}{c}\text { Cacique Cretan } \\
\text { Olga Benario } \\
\text { Eli Vive } \\
\end{array}$ & $\begin{array}{l}\text { São Jerônimo } \\
\text { da Serra }\end{array}$ \\
\hline COOPERTERRA & CPPS & $\begin{array}{c}\text { Rosa Luxemburgo } \\
\text { Liberdade Camponesa }\end{array}$ & Ortigueira, Imbaú \\
\hline COPRAN & CPPS & $\begin{array}{c}\text { Dorcelina Folador } \\
\text { Zumbí dos Palmares } \\
\text { Iraci Salete Strozake } \\
\text { Roseli Nunes } \\
\text { Santinho } \\
\text { Che Guevara } \\
\text { Eli Vive } \\
\text { Libertação Camponesa }\end{array}$ & Arapongas \\
\hline COOPERCAM & CPPS & $\begin{array}{c}\text { José Martí } \\
\text { Teixeirinha } \\
\text { Jose Heraldio }\end{array}$ & São Miguel de Iguaçu \\
\hline COAPRA & CPPS & Antonio Conselheiro & Pitanga \\
\hline COOPERMATE & CPPS & Antonio Conselheiro & Santa Maria do Oeste \\
\hline COOPERCONTESTADO & CPPS & Contestado & Bituruna \\
\hline COOCAMP & CPPS & João de Paula & Renascença \\
\hline COOPERAGRA & CPPS & José Arnaldo & Mari Luz \\
\hline COCAVI & CPPS & Zumbi dos Palmares & Jardim Alegre \\
\hline $\begin{array}{l}\text { COPROESTE } \\
\text { (antigua COAGRI) }\end{array}$ & CPPS & $\begin{array}{c}\text { Lenin } \\
\text { Ireno Alves dos Santos } \\
\text { Mao Tse-Tung } \\
\text { Padre Josimo } \\
\end{array}$ & Laranjeiras do Sul \\
\hline COPROTERRA & CPPS & $\begin{array}{c}\text { Lênin } \\
\text { Ireno Alves dos Santos } \\
\text { Mao Tse-Tung } \\
\text { Padre Josimo } \\
\end{array}$ & Laranjeiras do sul \\
\hline COTRAMIC & CPPS & Emiliano Zapata & Castro (Ponta Grossa) \\
\hline COOPERJUNHO & CPS & $\begin{array}{c}\text { Lenin } \\
\text { (Assentamento } \\
8 \text { de Junho) }\end{array}$ & Laranjeiras do Sul \\
\hline $\begin{array}{l}\text { COOPERATIVA } \\
\text { TERRA LIVRE }\end{array}$ & CPS & $\begin{array}{c}\text { Monje Jozé Maria } \\
\text { (Assentamento Contestado) }\end{array}$ & Lapa \\
\hline COPAVI & $\mathrm{CPA}$ & $\begin{array}{c}\text { Iraci Salete Stronzake } \\
\text { (Assentamento } \\
\text { Santa Maria) }\end{array}$ & Paranacity \\
\hline IRMA DOROTHY (APROID) & Asociación & $\begin{array}{c}\text { Rosa Luxemburgo } \\
\text { (Assentamento Guanabara) }\end{array}$ & Imbaú \\
\hline $\begin{array}{l}\text { CCA/PR } \\
\text { Cooperativa Central }\end{array}$ & CCA & Paraná & Curitiba \\
\hline CREHNOR & Crédito & MST & Laranjeiras do Sul \\
\hline
\end{tabular}


Figura 3. Distribución territorial de las cooperativas del MST en relación a las Brigadas. Estado de Paraná, 2013.

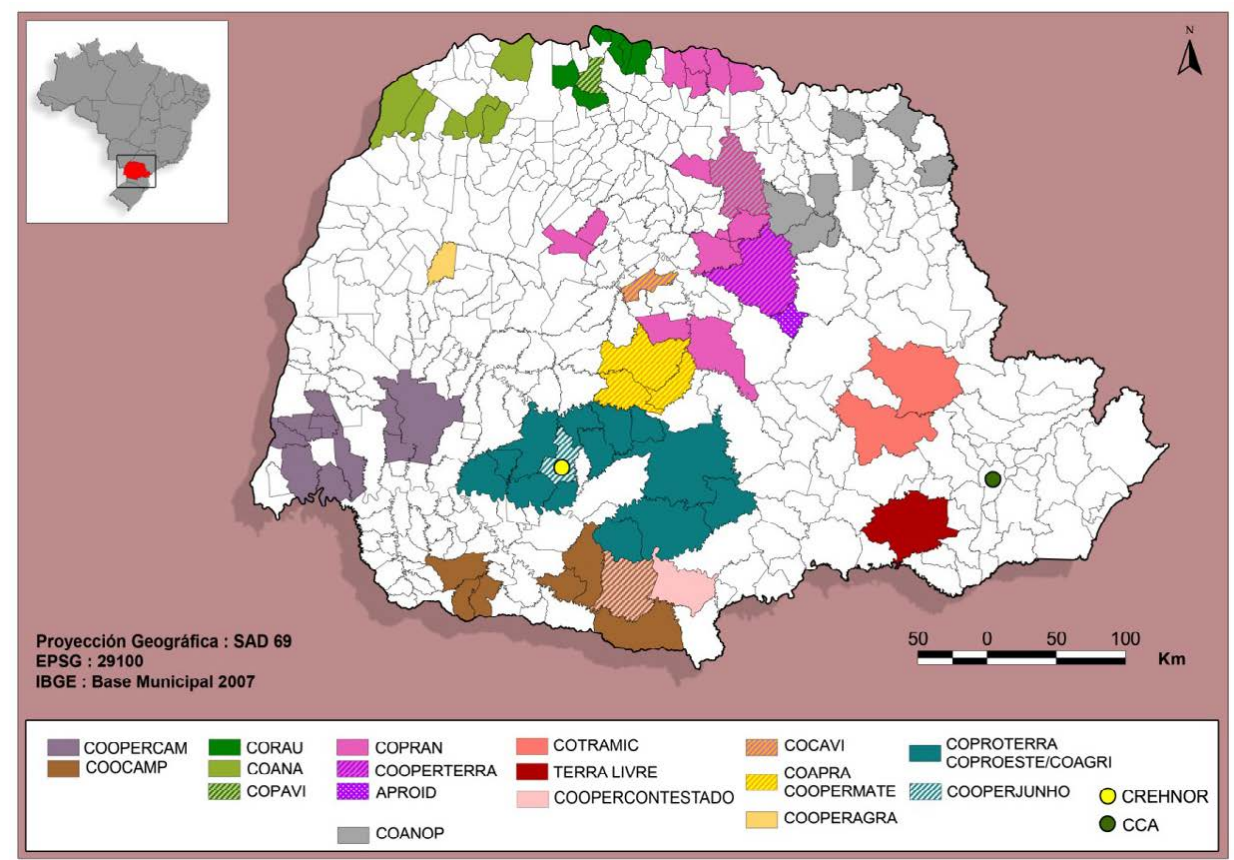

Elaboración propia

Las cooperativas del Movimiento Sin Tierra organizadas en el estado de Paraná se presentan como hitos territoriales en la construcción de dinámicas de producción basadas en la agricultura familiar campesina, la cooperación agrícola, la agroecología, y la intercoopreción cooperativa. Las familias integradas en las Cooperativas de Producción y Prestación de Servicios (CPPS) se distribuyen por líneas de producción, principalmente de arroz, lácteos, azúcar, mate, frutas y verduras (hortofrutícolas), y miel; dedicadas especialmente a la fase de industrialización y comercialización de productos de reforma agraria, y coordinadas por el Sector de Producción. Todas estas cooperativas están registradas legalmente en la junta comercial, y por su vocación eminentemente económica están expuestas a exigentes medidas de control fiscal y gubernamental.

Mediante el trabajo de campo se verifica que no todos los Núcleos de Producción poseen una implicación real con la práctica de un trabajo cooperativo, aunque la planificación cooperativa de la producción y la asistencia técnica sí está garantizada por la misma. De igual forma, las posibilidades de acceso a crédito, la obtención de capital y la política de inversiones están centralizadas conjuntamente a través de sus propias cooperativas ${ }^{17}$. La dimensión de las cooperativas es muy variable y todavía inestable ya que el proceso de consolidación de las mismas dependerá en gran medida de la capacidad de desarrollar dinámicas de autogestión y de viabilidad económica suficiente para su continuidad. En este contexto, más de medio centenar de cooperativas del MST accedieron a la última fase del programa gubernamental Terra Forte, de las cuales tres cooperativas del estado de Paraná serán financiadas durante los próximos años para el desarrollo de sus agroindustrias: COOPERMATE, para la producción de hierba mate y derivados, CORAU, sector del azúcar de caña y leche, y COOPERJUNHO, leche, horticultura y panificados.

En todos los assentamentos se producen aquellos productos tradicionalmente vinculados a la agricultura campesina (frijoles y mandioca), además de una gran variedad de hortalizas y frutas adaptadas a las condiciones territoriales de cada región. Por consiguiente, son los núcleos de base y/o de producción de los assentamentos, o sea la escala local, la principal escala donde territorializar la cooperación y la ayuda mutua en la esfera de la producción.

17 Dependiendo del programa y la necesidad de las familias se usan las asociaciones o las cooperativas para acceder a créditos, ayudas y programas de inversión. Si se trata de solicitudes de mejora de los assentamentos (casas, escuelas, centro médico, etcétera), son las asociaciones las que administran esta función. Si las mejoras, en cambio, atañen directamente al proceso productivo y de trabajo, son las cooperativas las que asumen esta articulación. 
Tabla 2. Base socioeconómica y territorial de las cooperativas del MST. Estado de Paraná, 2013.

\begin{tabular}{|c|c|c|c|c|}
\hline COOPERATIVA & $\begin{array}{l}\text { Número de } \\
\text { assentamentos }\end{array}$ & $\begin{array}{l}\text { Personas } \\
\text { asociadas }\end{array}$ & $\begin{array}{l}\text { Producto } \\
\text { principal }\end{array}$ & Producción secundaria \\
\hline CORAU & 9 & 107 & Caña de azúcar & Hortalizas y lácteos \\
\hline COANA & 17 & 1.605 & Arroz & Lácteos \\
\hline COANOP & 24 & 656 & Café & Insumos \\
\hline COOPERTERRA & 19 & 620 & Semillas criollas & Hortalizas \\
\hline COPRAN & 24 & 428 & Lácteos & Hortalizas \\
\hline COOPERCAM & 9 & 430 & Hortalizas & Pan \\
\hline COAPRA & 4 & 186 & Lácteos & Hortalizas y frutas \\
\hline COOPERMATE & 5 & $22(598)^{*}$ & Hierba mate & Derivados del mate \\
\hline COOPERCONTESTADO & 7 & 151 & Miel & Hortalizas y frutas \\
\hline СООСАМР & 3 & 223 & Lácteos & Hortalizas y frutas \\
\hline COOPERAGRA & 1 & - & Hortalizas & - \\
\hline COCAVI & 1 & 112 & Lácteos & Hortalizas y frutas \\
\hline $\begin{array}{l}\text { COPROESTE } \\
\text { (antigua COAGRI) }\end{array}$ & - & - & Trabajo & Servicios \\
\hline COPROTERRA & - & - & Lácteos & - \\
\hline COTRAMIC & 3 & 180 & Lácteos & - \\
\hline COOPERJUNHO & 1 & 44 & Pan y dulces & Hortalizas \\
\hline COOP. TERRA LIVRE & 1 & 205 & Hortalizas y frutas & - \\
\hline COPAVI $* * *$ & 1 & 35 & Caña de azúcar & Lácteos, Hortalizas y frutas \\
\hline IRMA DOROTHY (APROID)*** & 1 & 12 & Semillas criollas & - \\
\hline CREHNOR & 16 (municipios) & $4.200^{* *}$ & Créditos y seguros & - \\
\hline CCA/PR & \multicolumn{4}{|c|}{$\begin{array}{l}\text { Representación política e institucional. } \\
\text { Función comercial y de servicios. }\end{array}$} \\
\hline Total & 128 & 5.592 & & \\
\hline
\end{tabular}

Elaboración propia

De modo que, para el MST los assentamentos reflejan aquellos lugares "a partir de los cuales organizar el trabajo colectivo y desarrollar su función económica para las familias" (MST, 2005: 93). Y, particularmente, para Neuri Rosseto ${ }^{18}$ el assentamento ha de convertirse en la "carta de visita de una nueva agricultura, una agricultura caracterizada por la producción cooperativa, agroecológica y socialista”. Para ello, en el Encuentro Estadual del MST en Paraná celebrado en diciembre de 2012 y ratificado en el VI Congreso Nacional de 2014, la nueva geoestrategia del movimiento pasa por la reivindicación de una Reforma Agraria Popular que conlleva la aplicación de políticas públicas de distribución de tierras, una inversión en la agricultura familiar, un compromiso social y político por generar y consolidar otras formas de producir y comercializar productos de forma cooperativa, y en la defensa de la soberanía alimentaria y la agroecología; en definitiva, se establece una concepción de desarrollo territorial confrontada directamente con la propuesta capitalista del agronegocio. 


\subsection{La comercialización cooperativa de los productos de reforma agraria}

Tanto en la esfera de la producción como en la comercialización se establecen relaciones de intercooperación territorial entre cooperativas del MST vinculadas a las diferentes líneas de producción. Entre ellas destaca la diversidad de producción generada por las cooperativas paranaenses como el sector de leche y derivados (yogurt y queso), café, arroz, hierba mate, caña de azúcar y derivados (melado o cachaça), miel, así como el papel estratégico de producción de semillas criollas autóctonas, centralizada en la cooperativa COOPERTERRA. En el Movimiento Sin Tierra existen, además, otras cooperativas dedicadas a la gestión de trabajos y servicios que actúan en diferentes estados, como COPROESTE (Laranjeiras do Sul) o COOPERAR, cuya sede social se encuentra en São Paulo.

En el estado de Paraná se ha consolidado especialmente la producción de lácteos y derivados, como los quesos y el yogurt "Campo Vivo", marca comercial de cooperativas COANA y COPRAN, que centralizan la producción agroindustrial de lácteos en todo el estado. Estas cooperativas se encargan de la recogida de materia prima (leche) de las diversas familias localizadas en 48 assentamentos diferentes, así como de ofrecer asistencia técnica, y controles de calidad y seguridad alimentaria para su comercialización. De igual forma, otras cooperativas como TERRA LIVRE o COOPERCAM destacan por la consolidación de una producción completamente agroecológica de frutas y verduras, así como por una mayor organización en la esfera de la comercialización y distribución.

Figura 4. Líneas principales de producción de las cooperativas del MST. Estado de Paraná (Brasil), 2013.

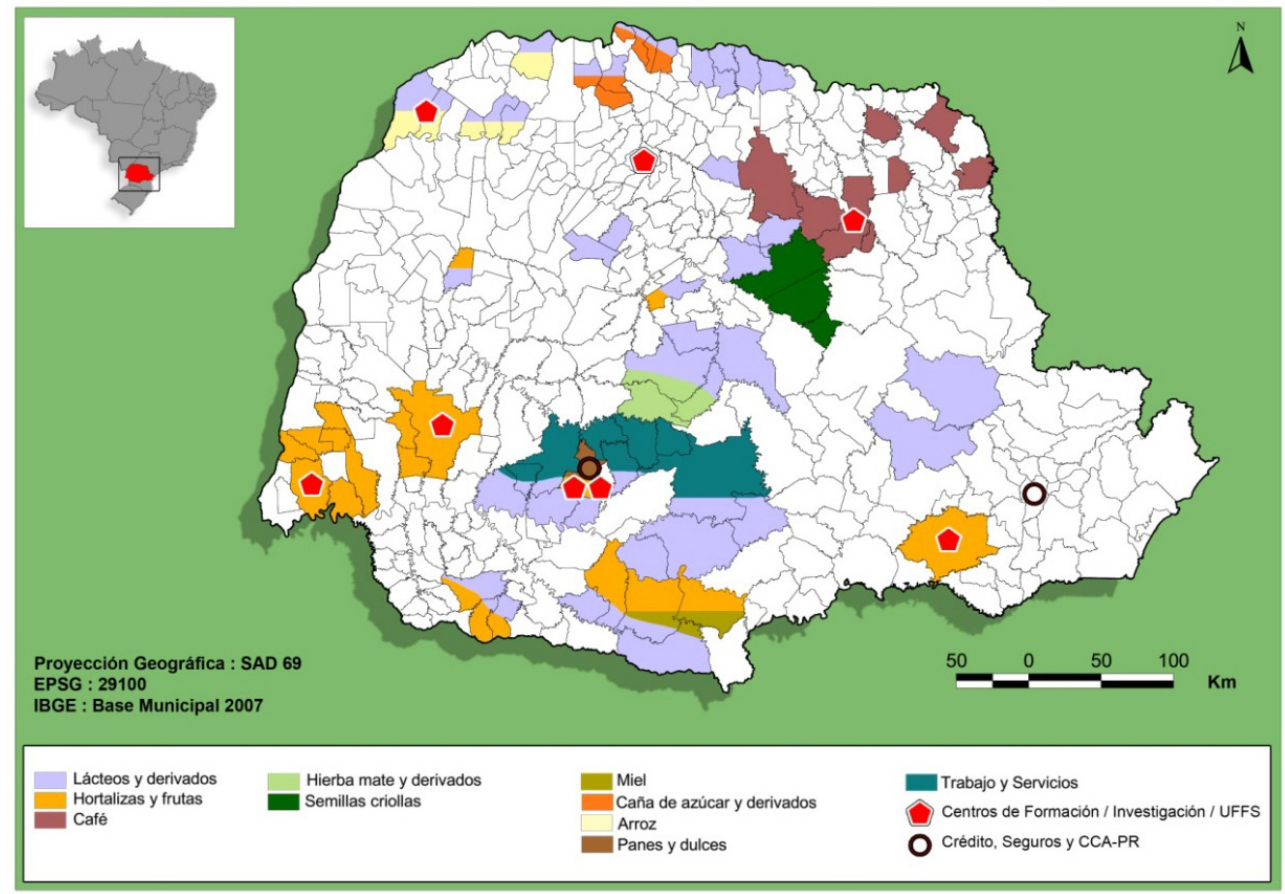

Elaboración propia

La comercialización de los productos de reforma agraria producidos por las familias assentadas del MST presenta una serie de insuficiencias estructurales y organizativas importantes. Por un lado, debido a las numerosas dificultades aún vigentes en la organización productiva de los assentamentos y de las familias, las vías de comercialización de la producción familiar son la venta directa (mercadillos y ferias populares), la venta mediante algún intermediario (empresas privadas de distribución minorista o mayorista), a través de asociaciones propias de cada assentamento o, más recientemente, a través de las cooperativas CPPS o bien desde la Cooperativa Central. En el caso de la COPAVI (una CPA), la propia cooperativa integra todo el proceso de distribución y comercialización, y ha desarrollado, además, particulares formas de distribución como la venta directa en casas del municipio en el que se localizan (Paranacity). Algunas cooperativas, incluso, exportan al extranjero los productos con la marca "Productos da Terra" del MST (COPERMATE y también COPAVI). 
Es preciso indicar que prácticamente todas las cooperativas destinan sus productos de reforma agraria a los programas gubernamentales como el Programa de Adquisición de Alimentos (PAA) y el Programa Nacional de Alimentación Escolar (PNAE), destinados a abastecer los comedores de escuelas, guarderías, hospitales, centros sociales o ayuntamientos; lo que vendría a denominarse el "mercado institucional". Son programas que se adjudican anualmente a aquellos emprendimientos de la agricultura familiar (asociaciones y cooperativas) para la adquisición de alimentos de reforma agraria ${ }^{19}$, un mercado que actualmente supone la salida fundamental para la producción de las cooperativas agrarias del MST. No obstante, el abastecimiento de este mercado institucional suscita una serie de cuestiones relevantes acerca del nivel de dependencia que tienen las cooperativas del MST respecto a los programas públicos de adquisición de alimentos, que en algunos casos supone casi el total de la producción. Los canales comerciales utilizados para la venta de sus productos son los mercados locales y ferias de productores, pequeños comercios, supermercados locales y los antiguos supermercados Coopera, instalados en la Comunidad de Arapongas, assentamento "Ireno Alves dos Santos" en Rio Bonito do Iguaçu; y en el municipio Nova Laranjeiras, a lo que es preciso añadir la distribución mediante algunas grandes cadenas de distribución y centros comerciales privados, lo cual ha supuesto un debate importante sobre las contradicciones a las que se enfrenta el movimiento.

Entre los canales de distribución y consumo de productos de reforma agraria elaborados por el MST también es preciso contabilizar el papel de las escuelas y centros de formación del movimiento, y también sus secretarías estaduales, estrechamente relacionadas a las cooperativas regionales (CPPS) y dedicadas a la formación política y técnica de su base social, abastecidas de hecho por los assentamentos más cercanos. La red de centros de formación del MST en Paraná, que acompaña la territorialización cooperativa y campesina, está compuesta por: el Centro de Desarrollo Sostenible y Capacitación en Agroecología (CEAGRO) en Laranjeiras do Sul, donde se realiza un Curso en Tecnología y Gestión de Cooperativas -TGC- desde 2010; el Instituto Técnico de Educación e Investigación de la Reforma Agraria (ITEPA) en São Miguel de Iguaçu; la Escola Milton Santos (EMS) en la ciudad de Maringá; la Escuela Latinoamericana de Agroecología (ELAA) en el municipio de Lapa, cerca de la capital Curitiba; el Centro de Estudios y Formación Che Guevara (CEFAG) en Querência do Norte; la Casa Familiar Rural-Padre Sasaki (CRF) en Congonhinhas, región norte; el Centro de Enseñanza e Investigación en Agroecología Valmir Mota de Oliveira-Keno en Cascavel; y también la Universidad Federal Frontera Sul, la primera y única universidad situada en el interior de un assentamento de reforma agraria del MST, en este caso en el assentamento " 8 de Junho", en el término municipal de Laranjeiras do Sul. A dicha red habría que agregar la Escola Nacional Florestan Fernandes del MST (ENFF) localizada en el municipio de Guararema (estado de São Paulo) y el Instituto Técnico de Capacitación e Investigación de Reforma Agraria Josué de Castro -ITERRA-, situada en Veranópolis (Rio Grande do Sul) ${ }^{20}$.

Por el contrario, el MST en Paraná todavía no ha generado estructuras cooperativas autónomas suficientes en la esfera de la distribución. A pesar de ello, se están impulsando algunas dinámicas e iniciativas alternativas como la red de producción agroecológica bajo el certificado de Rede Ecovida ${ }^{21}$. Y, por ejemplo, en la ciudad de Curitiba, capital de Paraná, se han iniciado articulaciones de circuitos cortos de comercialización ligados directamente con grupos de consumo urbano, para cubrir la demanda de profesores, asociaciones y organizaciones sensibilizadas con el consumo responsable y el consumo de productos de reforma agraria.

Los desafíos del movimiento en la esfera de la comercialización se encuentran, no obstante, en la implantación de tres medidas fundamentales: En primer lugar, avanzar en la oportunidad de abastecer a las mismas familias del MST, a través de la distribución en sus propios assentamentos y tiendas, de productos producidos por el MST, mediante sus cooperativas y/o por otras cooperativas del movimiento. Asimismo, intentar abastecer a todas las escuelas y secretarías políticas del Movimiento Sin Tierra. Supone una

19 La Ley 11947/09 establece que las ayudas gubernamentales dotadas al abastecimiento de alimentos para las instituciones públicas, sobre todo la red de escuelas públicas, se deben destinar a la compra de al menos un 30\% de los alimentos de procedencia de la agricultura familiar (AF), aplicando una serie de mecanismos de garantía de precios.

20 En el ITERRA se organiza el curso de Técnico en Administración de Cooperativas (TAC) desde 1993, formando así a algunos de los administradores actuales de las cooperativas paranaenses del MST, como el caso de la cooperativa COANOP, en la región norte pionero de Paraná.

$21 \mathrm{La}$ Red de Agroecología ECOVIDA se estructura en núcleos de agricultores familiares, técnicos y consumidores para el desarrollo de la agroecología. Consolidada sobre todo en la región sur de Brasil. Fuente on-line: http://www.ecovida.org.br/ [Última consulta, diciembre de 2013]. 
gran contradicción observar la carencia de productos propios del movimiento en el consumo cotidiano de las familias assentadas, lo que significaría un mercado potencial que ha sido escasamente atendido. En segundo lugar, la creación de tiendas y supermercados propios del MST, así como la instalación de locales propios en centros urbanos cercanos; que iría unido al proceso de consolidación de las marcas de identidad del movimiento como son "Campo Vivo" o "Produtos da Terra". Y en tercer lugar, la apuesta por una intercooperación comercial (intercambios) entre cooperativas de la Vía Campesina de productos no perecederos como café, arroz, hierba mate, vino, aceites, dulces u otros.

En última instancia, la generalización de una intercooperación entre cooperativas de producción en el seno del MST, con intercambios entre sí, y de éstas con otras cooperativas de consumo o asociaciones de consumo; a lo que cabría añadir el ejercicio de una intercooperación cooperativa entre el medio rural y el medio urbano; todo ello impulsaría nuevas dinámicas hacia la transformación social y espacial mediante la territorialización cooperativa y campesina.

\section{CONCLUSIONES}

Como movimiento socioterritorial, el MST ha asumido, junto con todas las organizaciones integradas en la Vía Campesina, la lucha por una soberanía alimentaria de los pueblos y la generalización de una estructura cooperativa y una matriz tecnológica agroecológica de producción. En este sentido, la transcendencia de la cooperación agrícola en el MST en el estado de Paraná no se ciñe únicamente a la creación aleatoria de cooperativas, sino la puesta en práctica de una estrategia de transformación socioespacial mediante la práctica cooperativista, en base a pautas de trabajo cooperativo y de ayuda mutua organizadas en Brigadas. Es decir, una territorialización cooperativa y campesina en su sentido más amplio, estrechamente vinculada a la organización social y política del movimiento. Por lo que las cooperativas del MST no se pueden analizar de forma aislada, sino integradas en un proyecto político más ambicioso y contextualizadas en las respectivas redes de intercooperación a diferentes escalas, incluida la internacional.

En esta propuesta queda patente la integración de los valores cooperativos en los diferentes ámbitos de la vida cotidiana y en las distintas esferas del proceso productivo, válidas tanto para el trabajo en el campo como para la ciudad. Sin embargo, todavía existen insuficiencias evidentes de organización cooperativa en la esfera de la distribución y del consumo de los productos de reforma agraria.

En el conjunto de esta dinámica de territorialización protagonizada por las familias del MST destaca, por ende, la práctica de una agricultura familiar campesina y la organización de un trabajo cooperativo; la elaboración de un producto saludable y de calidad; la producción exclusivamente agroecológica y diversificada; la búsqueda de mercados locales, un consumo de proximidad y de confianza; el diseño de programas de formación política y capacitación técnica a través de sus propias escuelas de educación y formación; la reivindicación de una redistribución social de la riqueza, y todo un compendio de trabajo en red y de intercooperación solidaria internacionalista.

La reforma agraria popular promovida durante el VI Congreso del MST en febrero de 2014 recoge y consolida los horizontes de una territorialización del trabajo cooperativo en el movimiento campesino ligados a la conciencia de clase, con la finalidad de impulsar una transformación del espacio geográfico y, por tanto, de la sociedad.

\section{REFERENCIAS}

BOGO, A. (2002): O vigor da mistica. Caderno de Cultura n²,São Paulo:MST, 189 pp.

CALDART, R. S. (2004): Pedagogia do Movimento Sem Terra. $3^{a}$ ed. São Paulo: Expressão Popular, 439 pp.

CALLE, A. (2002): "Análisis comparado de movimientos sociales: MST, Guatemala y España". Revista Terra Livre: Associação dos Geógrafos Brasileiros, nº 19, vol. 2. São Paulo: AGB, pp.37-58.

CARBALLO, M. (2011): Vem, teçamos a nossa liberdade: Mujeres líderes en el Movimiento Sin Tierra, Ceará (Brasil). Dirección: Verena Stolcke. Tesis de doctorado en antropología social, cultural y etnografía. Universitat Autònoma de Barcelona, 563 pp.

CHAYANOV, Alexander V. (1985): La organización de la unidad económica campesina. Buenos Aires: Nueva visión, $342 \mathrm{pp}$. 
CONCRAB (1995): Perspectivas da cooperação agrícola nos assentamentos. Caderno de Cooperação Agricola, $\mathrm{n}^{\circ}$ 4. São Paulo: MST, 17 pp.

CONCRAB (1996): Cooperativas de produção: questões práticas. Caderno de formação, nº 21, São Paulo: MST-Concrab, 89 pp.

CONCRAB (1998): Sistema Cooperativista dos assentados. Caderno de cooperação agrícola, nº 5. São Paulo: MST, 76 pp.

CONCRAB (2000): O cooperativismo no pensamento marxista. Caderno das experiências historicas da cooperação, $\mathrm{n}^{\circ}$ 2. São Paulo: Concrab, 100 pp.

CONCRAB (2004a): A constituição e o desenvolvimento de formas coletivas de organização e gestão do trabatho em assentamentos de reforma agrária. Caderno de cooperação agrícola, $\mathrm{n}^{\circ} 11$. São Paulo: Concrab, $93 \mathrm{pp}$.

CONCRAB (2004b): O agronegócio x Agricultura familiar e a reforma agrária. Brasilia D. F: Concrab, 103 pp.

FERNANDES, B. M. (2005): "Movimentos socioterritoriais e movimentos socioespaciais". Observatorio Social de América Latina., v.16, Buentos Aires: CLACSO, pp. 273-284.

GIRIBET BERNAT, I. (2012): Um novo jeito de organizar. A Nova Organicidade em/do Movimento; Luta pela terra, Luta na terra e Identidade Sem Terra na área de influência da Brigada Salvador Allende-MST (Paraná, Brasil). Dirección: D. Jaume Barrull Pelegrí y D. Antonio Thomaz Júnior. Tesis doctoral en Historia. Universitat de Beida, 292 pp.

HARNECKER, M. (2002): Sin Tierra. Construyendo Movimiento Social. Madrid: Siglo XXI, 303 pp.

HOLT-GIMÉNEZ, Eric; PEABODY, Loren (2008): "De las revueltas del hambre a la soberanía alimentaria: Un llamado urgente para reconstruir el sistema agroalimentario". En VV AA: Introducción a la crisis agroalimentaria global. Barcelona: Grain, Entrepueblos, No et mengis el món, VSF, ODG, Xarxa Consum Solidari, 6-15 p.

MARTIN, J-Y. (2001): Les Sans-Terre du Brésil. Géographie d’un mouvement socio-territorial. Paris: Harmattan, $173 \mathrm{pp}$.

MARX, K. (1975a): "Salario, precio y ganancia". En: Marx, K. y Engels, F:: Obras Escogidas. Madrid: Akal. Vol.1, pp. 410-465.

MARX, K. (1975b): "Trabajo asalariado y capital". En: Marx, K. y Engels, F: Obras Escogidas. Madrid: Akal. Vol.1, pp. 71-99.

MST (2002): Pedagogia da terra. Cuadernos ITERRA, n². Vereanópolis, RS:MST, 98 pp.

MST (2005): Método de trabalho e organização popular. Setor Nacional de Formação. São Paulo: MST, 170 pp.

MST (2008a): Programa de Formação para a Cooperação e Organização dos Assentamentos. Cartilha de Apoio $n^{\circ}$ 1. São Paulo: MST, 96 pp.

MST (2008b): Programa de Formação para a Cooperação e Organização dos Assentamentos. Cartilha de Apoio $\mathrm{n}^{\circ}$ 2. São Paulo: MST, $186 \mathrm{pp}$.

SANTOS, M (2009a): A natureza do espaço: técnica e tempo, razão e emoção. $4^{a}$ ed. São Paulo: Edusp, 388 pp. [1996].

STÉDILE, J. P. (Org.) (2002): História e natureza das ligas camponesas. São Paulo: Expressão popular, 190 pp.

STÉDILE, J. P.; FERNANDES, B. M. (1999): Brava gente: A trajetoria do MST e a luta pela terra no Brasil. São Paulo: Fundação Perseu Abramo, 168 pp. 